九州大学学術情報リポジトリ

Kyushu University Institutional Repository

\title{
BAHADUR REPRESENTATION OF SAMPLE CONDITIONAL QUANTILES BASED ON SMOOTHED CONDITIONAL EMPIRICAL DISTRIBUTION FUNCTION
}

Mehra, K. L.

Department of Statistics and Applied Probability, University of Alberta

Krishnaiah, Y. S. Rama

Osmania University

Rao, M. Sudhakara

Osmania University

https://doi.org/10.5109/13425

出版情報: Bulletin of informatics and cybernetics. 25 (1/2), pp.99-107, 1992-03. Research Association of Statistical Sciences

バージョン :

権利関係 : 


\title{
BAHADUR REPRESENTATION OF SAMPLE CONDITIONAL QUANTILES BASED ON SMOOTHED CONDITIONAL EMPIRICAL DISTRIBUTION FUNCTION*
}

By

\author{
K.L. Mehra, Y.S. Rama Krishnaiah** and M. Sudhakara RaO**
}

\begin{abstract}
Let $\left\{\left(X_{i}, Y_{i}\right): i=1,2, \ldots\right\}$ be a sequence of stationary independent random vectors in $\mathscr{R}^{(2)}$ with a continuous distribution, and let $G_{x}(\cdot)$ denote the conditional distribution function of $Y_{1}$ given $X_{1}=x$. In this paper, Bahadur's almost sure representation for the sample conditional quantile $\widehat{G}_{n x}^{-1}(\lambda), 0<\lambda<1$, is established, where $\widehat{G}_{m x}$ is a smoothed (rank nearest neighbor or the Nadaraya-Watson type) estimator of $G_{x}$. Such representations are useful in the study of asymptotics of functionals of conditional quantiles.
\end{abstract}

\section{Introduction}

Let $\left\{\left(X_{i}, Y_{i}\right), i=1,2, \ldots\right\}$ be a sequence of independent identically distributed random vectors with common continuous distribution function (d.f.) $H$ and marginal d.f.'s $F$ and $G$, respectively. Further, let $G_{x}$ denote the conditional d.f. of $Y$ given $X=$ $x \in \mathscr{R}=\mathscr{R}^{(1)}=\left(\right.$ real line) and, for each $\lambda, 0<\lambda<1$, let $q_{x}(\lambda)=G_{x}^{-1}(\lambda)=\inf \{y \in$ $\left.\Re: G_{x}(y) \geq \lambda\right\}$, the $\lambda^{\text {th }}$ quantile of $G_{x}$. Consider the smoothed conditional empirical d.f. defined by

$$
\begin{aligned}
\widehat{G}_{n x}(y) & =\left(n a_{n}\right)^{-1}\left(t_{n}(x)\right)^{-1} \sum_{i=1}^{n} W_{n}\left(a_{n}^{-1}\left(F_{n}(x)-F_{n}\left(X_{i}\right)\right), y-Y_{i}\right) \\
& =\left(a_{n} t_{n}(x)\right)^{-1} \iint W_{n}\left(\left(F_{n}(x)-F_{n}(u)\right) / a_{n}, y-v\right) d H_{n}(u, v),
\end{aligned}
$$

$-x<y<x$, where $H_{n}(x, y)=n^{-1} \sum_{i=1}^{n} I_{\left[X_{i} \leq x, Y, \leq y\right]}, F_{n}(x)=n^{-1} \sum_{i=1}^{n} I_{\left[X_{i} \leq x\right]},\left\{W_{n}\right\}$ is a "Heaviside" sequence as defined in Section 3 of Mehra, Rama and Rao [5] (hereafter abbreviated to MRR[5]) and $\left\{a_{n}\right\}$ is a bandwidth sequence $\left(a_{n} \downarrow 0\right.$ but $n a_{n} \rightarrow x$, as $n \rightarrow \infty)$. In this paper we shall establish a pointwise Bahadur [1] type almost sure representation for the sample $\lambda^{\text {th }}$ conditional quantile

Department of Statistics and Applied Probability, 434 Central Academic Building, University of Alberta. Edmonton, Canada T6G 2G1.

* The above research was supported in part by a CRF-NSERC Grant from the University of Alberta and the Grant No. A-3061 from the National Sciences and Enginecring Research Council of Canada

*** On leave from Osmania University, Hyderabad. India. 


$$
\widehat{G}_{n x}^{-1}(\lambda)=\inf \left\{y: \widehat{G}_{m x}(y) \geq \lambda\right\}, 0<\lambda<1,
$$

corresponding to the "smoothed" conditional empirical distribution function (s.c.e.d.f.) $\widehat{G}_{n x}$ defined by (1.1) and its Nadarya-Watson counterpart $\widehat{G}_{n x}^{*-1}(\lambda), 0<\lambda<1$ (see MRR [5] Section 1).

For unconditional unsmoothed sample quantile $G_{n}^{-1}(\lambda), 0<\lambda<1$, corresponding to the e.d.f. $G_{n}(y)$, Bahadur [1] showed that if $g\left(G^{-1}(\lambda)\right)>0$ and $g^{\prime}$ exists and is bounded in a neighbourhood of $G^{-1}(\lambda)$, then as $n \rightarrow \infty$, for each fixed $\lambda, 0<\lambda<1$, with probability one

$$
\begin{aligned}
G_{n}^{-1}(\lambda)-G^{-1}(\lambda) & \left.=-\left[g\left(G^{-1}(\lambda)\right)\right]^{-1}\left[G_{n}\left(G^{-1}(\lambda)\right)-\lambda\right)\right] \\
& +O\left(n^{-\frac{3}{4}}(\log n)^{\frac{1}{2}}(\log \log n)^{\frac{1}{4}}\right)
\end{aligned}
$$

(see also Kiefer [3]). Mack [4] extended the above a.s. representation result to sample quantiles $\widehat{G}_{n}^{-1}(\lambda)$ based on smoothed e.d.f. $\widehat{G}_{n}$ (see [4] Theorem pp. 187-188), but with the order term $O\left(n^{-\frac{3}{4}}(\log n)^{\frac{3}{4}}\right)$ converging to zero at a slightly slower rate as $n \rightarrow \infty$. In order to establish a similar a.s. representation for the conditional quantile (1.2) (Theorem 3.1. below), we need, in addition to Theorem 3.1 of MRR[5], some results regarding the oscillations of the $\widehat{G}_{m x}$. These results are given in Section 2 . The main result along with some remarks are given in Section 3. In order to avoid repetition, we employ the same notation and assumptions as used in MRR[5] and refer to the arguments therein whenever they help to shorten the proofs. Unlike as in MRR[5], the results of this paper are established explicitly only for the case $m=1$; the general case $m>1$ can be dealt with using similar arguments and the results of MRR[5] for the case $m>1$.

\section{Preliminary Results}

In this section, we shall prove two lemmas which are essential for the proof of the main result Theorem 3.1: Lemma 2.1 gives the a.s. rate of convergence of the smoothed conditional quantile $\widehat{G}_{n x}^{-1}(\lambda)$ to $G_{x}^{-1}(\lambda)$ and Lemma 2.2 pertains to the oscillation behaviour of the conditional empirical process. $\widehat{G}_{n x}(\lambda), 0<\lambda<1$. Let $\Lambda(F)$ denote the support of $F$.

LEMMA 2.1. Suppose that, in addition to the assumptions of Theorem 3.1(a) of $M R R[5], b_{n}^{2}=o\left(\tau_{n}\right)$, where $\left\{b_{n}\right\}$ is defined as in assumption A.MII(iv) of MRR[5]. Then for each $\lambda, 0<\lambda<1$ and each fixed $x \in \Lambda(F)$,

$$
\left[\widehat{G}_{n . x}^{-1}(\lambda)-G_{x}^{-1}(\lambda)\right]=O\left(\tau_{n}\right)
$$

with probability one, as $n \rightarrow \infty$, where $\widehat{G}_{n . x}^{-1}(\lambda)$ is defined by (1.2) and $\tau_{n}=n^{-\frac{1}{2}} a_{n}^{-\frac{1}{2}}$ $\left(\log a_{n}^{-1}\right)^{\frac{1}{2}}$.

Proof. First note that for each $\lambda, 0<\lambda<1$, and $\varepsilon>0$

$$
P\left[\bigcup_{k \geq n}\left\{\widehat{G}_{k x}^{-1}(\lambda)-G_{x}^{-1}(\lambda) \geq \varepsilon \tau_{k}\right\}\right]
$$




$$
\begin{aligned}
& =P\left[\bigcup_{k \geq n}\left\{\widehat{G}_{k x}\left(q_{x}(\lambda)+\varepsilon \tau_{k}\right) \leq \lambda\right\}\right]+P\left[\bigcup_{k \geq n}\left\{\widehat{G}_{k x}\left(q_{x}(\lambda)-\varepsilon \tau_{k}\right) \geq \lambda\right\}\right] \\
& =P\left[\bigcup_{k \geq n}\left\{\widehat{G}_{k x}\left(u_{k}^{+}\right) \leq \lambda\right\}\right]+P\left[\bigcup_{k \geq n}\left\{\widehat{G}_{k x}\left(u_{k}^{-}\right) \geq \lambda\right\}\right],
\end{aligned}
$$

where we have set $q_{x}(\lambda)=G_{x}^{-1}(\lambda), u_{n}^{+}=q_{x}(\lambda)+\varepsilon \tau_{n}$ and $u_{n}^{-}=q_{x}(\lambda)-\varepsilon \tau_{n}$. Now for the second term in (2.2), we obtain from (3.2) that WOLG (see (3.12a) of MRR[5]),

$$
\begin{aligned}
& P\left[\bigcup_{k \geq n}\left\{\widehat{G}_{k x}\left(u_{k}^{-}\right) \geq \lambda\right\}\right]=P\left[\bigcup_{k \geq n}\left\{t_{k}(x)\left(\widehat{G}_{k x}\left(u_{k}^{-}\right)-G_{k}\left(u_{k}^{-}\right)\right) \geq t_{k}(x)\left(\lambda-G_{x}\left(u_{k}^{-}\right)\right)\right\}\right] \\
& \leq P\left[\bigcup_{k \geq n}\left\{\left|\bar{J}_{k 1}\left(u_{k}^{-}\right)-E\left(\bar{J}_{k 1}\left(u_{k}^{-}\right)\right)-\left(t_{k 1}(x)-1\right)\right| \geq\left(\lambda-G_{x}\left(u_{k}^{-}\right)\right) / 2\right\}\right] \\
& +P\left[\bigcup_{k \geq n}\left\{\left|\sum_{j=2}^{3}\left(J_{k j}(y)+t_{k j}(x)\right)\right| \geq\left(\left(\lambda-G_{x}\left(u_{k}^{-}\right)\right) / 2\right)-E \bar{J}_{k 1}\left(u_{k}^{-}\right)\right\}\right]
\end{aligned}
$$

where $\tau_{n}=n^{-\frac{1}{2}} a_{n}^{-\frac{1}{2}}\left(\log a_{n}^{-1}\right)^{\frac{1}{2}}$ and $t_{n n}(x)=t_{n 1}(x)+t_{n 2}(x)+t_{n 3}(x)$ stands for the Taylor's expansion similar to that for $v_{n x}(y)$ in (3.2) of MRR[5]. Now note that, for the RHS expressions in the preceding probabilities in (2.3), we have by the mean value theorem, for some $\Delta_{n . x}$ lying between $G_{x}^{-1}(\lambda)$ and $\left(G_{x}^{-1}(\lambda)-\varepsilon \tau_{n}\right)$,

$$
\begin{aligned}
\tau_{\mathrm{n}}^{-1}\left[\lambda-G_{x}\left(u_{n}^{-}\right)\right] & =\tau_{\mathrm{n}}^{-1}\left[G_{x}\left(G_{x}^{-1}(\lambda)\right)-G_{x}\left(G_{x}^{-1}(\lambda)-\varepsilon \tau_{\mathrm{n}}\right)\right] \\
& =\varepsilon g_{x}\left(\Delta_{n x}\right) \\
& \rightarrow \varepsilon g_{x}\left(G_{x}^{-1}(\lambda)\right)>0,
\end{aligned}
$$

as $n \rightarrow \infty$, and further from (3.13) and (3.15) of MRR[5] and the additional assumption $b_{n}^{2}=\sigma\left(\tau_{n}\right)$, that

$$
E\left[\bar{J}_{n 1}\left(u_{n}^{-}\right)\right]=\sigma\left(\tau_{n}\right),
$$

as $n \rightarrow \infty$. From (2.4) and (2.5), it follows by the arguments used in the proof of Theorem 3.1, from (3.16) to (3.19), of MRR[5] that the first probability in (2.3) is dominated by

$$
\begin{aligned}
\sum_{k \geq n} P\left[\mid \bar{J}_{k 1}\left(u_{k}^{-}\right)-E \bar{J}_{k 1}\left(u_{k}^{-}\right)-\right. & \left.\left(t_{k 1}(x)-1\right) \mid \geq(\varepsilon / 2) \tau_{k} g_{x}\left(G_{x}^{-1}(\lambda)\right)\right] \\
\leq c_{1} \sum_{k \geq n}\left[k^{-2}\right] & \rightarrow 0
\end{aligned}
$$

as $n \rightarrow \infty\left(c_{1}\right.$ above is a positive constant). Also the second probability in (2.3) goes to zero by (3.24) of MRR[5](cf. Lemma 3.1). Thus, in view of (2.3) and (2.6), the second probability $P\left[\cup_{k \geq n}\left\{\widehat{G}_{k x}\left(u_{k}^{-}\right) \geq \lambda\right\}\right]$ on the right of $(2.2) \rightarrow 0$, as $n \rightarrow \infty$. By following parallel arguments, one can similarly show that the first term in (2.2)

$$
P\left[\bigcup_{k \geq n}\left\{G_{k x}\left(u_{k}^{+}\right) \leq \lambda\right\}\right] \rightarrow 0,
$$

as $n \rightarrow x$. The proof of the Lemma accordingly follows from (2.2) and the standard a.s. 
convergence criterion.

COROLlary 2.1. Under the conditions of Lemma 2.1, for each $\lambda, 0<\lambda<1$, $\widehat{G}_{n x}^{-1}(\lambda)$ is a strongly consistent estimator of $q_{x}(\lambda)=G_{x}^{-1}(\lambda)$, as $n \rightarrow \infty$.

The following lemma gives the order of magnitude of oscillations of the conditional empirical process based on $\widehat{G}_{n x}(y), y \in \Re$.

Lemma 2.2. Suppose the conditions of lemma 2.l hold. Then, for each $\lambda, 0<\lambda<$ 1 , and any fixed $x \in \Lambda(F)$ and constant $c>0$, we have with probability one

$$
\sup _{\left|y-q_{1}(\lambda)\right|<\tau_{n}}\left|\widehat{G}_{n . x}(y)-\widehat{G}_{n . x}\left(q_{x}(\lambda)\right)-G_{x}(y)+\lambda\right|=O\left(\tau_{n}^{3 / 2}\right),
$$

as $n \rightarrow \infty$, where $\tau_{n}$ is as defined in Lemma 2.1.

Proof. For each fixed $\lambda, 0<\lambda<1$, and $x \in \Lambda(F)$, consider the real numbers $\eta_{r n}$ defined by

$$
\eta_{r n}=q_{x}(\lambda)+r \tau_{n}^{*}, r=0, \pm 1, \ldots \pm d_{n},
$$

where $c>0$ is a constant, $\tau_{n}^{*}=c \tau_{n}^{3 / 2}, d_{n}=\left[\tau_{n}^{-\frac{1}{2}}\right]+1$, and $[\cdot]$ stands for the integral part. Then by the monotonicity of $\widehat{G}_{n x}$ and $G_{x}$, we have for any $y \in I_{r}=\left[\eta_{r n}, \eta_{(r+1) n}\right]$,

$$
\begin{aligned}
\widehat{G}_{n \cdot x}\left(\eta_{r n}\right)-G_{x}\left(\eta_{(r+1) n}\right) & \leq \widehat{G}_{n x}(y)-G_{x}(y) \\
& \leq \widehat{G}_{n x}\left(\eta_{(r+1) n}\right)-G_{x}\left(\eta_{r n}\right),
\end{aligned}
$$

which yields

$$
\begin{aligned}
& \sup _{\left|y-q_{x}(\lambda)\right|<c \tau_{n}}\left|\widehat{G}_{n x x}(y)-G_{x}(y)-\widehat{G}_{n x}\left(q_{x}(\lambda)\right)+\lambda\right| \\
& \leq \max _{|r| \leq d_{n}}\left|\widehat{G}_{n x x}\left(\eta_{r n}\right)-G_{x}\left(\eta_{r n}\right)-\widehat{G}_{n \cdot x}\left(q_{x}(\lambda)\right)+\lambda\right| \\
& +\max _{-d_{n} \leq r \leq d_{n}-1}\left|G_{x}\left(\eta_{(r+1) n}\right)-G_{x}\left(\eta_{r n}\right)\right| .
\end{aligned}
$$

By assumption A.I(ii), the density $g_{x}$ is bounded (in some neighborhood of $q_{x}(\lambda)$ ) so that for sufficiently large $n$ the second term on the R.H.S. in (2.10) is bounded by $\tau_{n}^{*}$. It thus suffices to show that the first term on the R.H.S. of (2.10) is dominated by an order term given in the assertion of the lemma. For this, note that from (3.2) of MRR[5] we have

$$
\begin{aligned}
& t_{n}(x)\left[\widehat{G}_{n x}\left(\eta_{r n}\right)-\widehat{G}_{n x}\left(q_{x}(\lambda)\right)-G_{x}\left(\eta_{r n}\right)+\lambda\right] \\
& \quad=\left[J_{n 1}\left(\eta_{r n}\right)-J_{n 1}\left(q_{x}(\lambda)\right)\right]+\left[J_{n 2}\left(\eta_{r n}\right)-J_{n 2}\left(q_{x}(\lambda)\right)\right]+\left[J_{n 3}\left(\eta_{r n}\right)-J_{n 3}\left(q_{x}(\lambda)\right)\right] \\
& \quad=\left[\varepsilon_{n 1}+\varepsilon_{n 2}+\varepsilon_{n 3}\right] \text { (say). }
\end{aligned}
$$

We first deal with the terms $\varepsilon_{n 2}$ and $\varepsilon_{n 3}$. Now as in Lemma 3.1 of MRR[5], we have from (3.4), (3.5) and (3.6) of MRR[5], with probability one and uniformly in $r$, for some constants $C_{5}$ and $C_{6}$ 


$$
\begin{aligned}
\left|\varepsilon_{n 3}\right| & \leq \varepsilon_{n 31}+\varepsilon_{n 32} \\
& \leq C_{5} n^{-\frac{3}{2}} a_{n}^{-2} \log a_{n}{ }^{-1}(\log \log n)^{\frac{1}{2}}+C_{6} n^{-1} a_{n}^{-1} \log a_{n}^{-1} \\
& =\left(n^{-1} a_{n}^{-1} \log a_{n}^{-1}\right)^{\frac{3}{4}}\left[C_{5} n^{-\frac{3}{4}} a_{n}^{-\frac{5}{4}}\left(\log a_{n}^{-1}\right)^{\frac{1}{4}}(\log \log n)^{\frac{1}{2}}+C_{6} n^{-\frac{1}{4}} a_{n}^{-\frac{1}{4}}\left(\log a_{n}^{-1}\right)^{\frac{1}{4}}\right] \\
& =\sigma\left(\tau_{n}^{\frac{3}{2}}\right)
\end{aligned}
$$

as $n \rightarrow \infty$, under the assumption A.IV(ii). Now splitting $\varepsilon_{n 2}$, on exactly the same lines as for $J_{n 2}$ in (3.8) of MRR[5], we obtain

$$
\varepsilon_{n 2}=\varepsilon_{n 21}+\varepsilon_{n 22} \quad(\text { say })
$$

where, in view of boundedness of $W_{n}$ and $W_{n}^{*}$ (cf (3.9) of MRR[5]), we have uniformly in $r$

$$
\begin{aligned}
\left|\varepsilon_{n 22}\right| & \leq C_{7}\left(n^{-1} a_{n}^{-1} \log a_{n}^{-1}\right)^{3 / 4} n^{-\frac{1}{4}} a_{n}^{-3 / 4}(\log \log n)^{\frac{1}{2}}\left(\log a_{n}^{-1}\right)^{-\frac{1}{4}} \\
& \left.=C_{7} \tau_{n}^{3 / 2}\left(n^{-1} a_{n}^{-3}\right)^{1 / 4}\left[(\log \log n)^{2} / \log a_{n}^{-1}\right)\right]^{1 / 4} \\
& =\sigma\left(\tau_{n}^{3 / 2}\right) \\
& \text { a.s. }
\end{aligned}
$$

as $n \rightarrow \infty$, in view of assumptions A.IV(ii); further on the lines of (3.10) of MRR[5], we have

$$
\begin{aligned}
\left|\varepsilon_{n 21}\right| \leq & \tau_{n}\left[\int_{A_{n}}\left|W_{n}^{*}(1)(t)\right|\left|\int\left[V_{n}^{(1,0)}\left(t, \eta_{r n}-v\right)-V_{n}^{(1,0)}\left(t, q_{x}(\lambda)-v\right)\right] d G_{x}(v)\right| d t\right. \\
+ & \left.\left(\int\left|W_{n}^{*(1)}(t)\right| d t\right) \mid G_{x}\left(\eta_{r n}\right)-\lambda\right) \mid \\
& +\iint_{A_{n}^{*}}\left|W_{n}^{(1.0)}\left(t, \eta_{r n}-v\right)-W_{n}^{(1.0)}\left(t, q_{x}(\lambda)-v\right)-W_{n}^{(1)^{*}}(t)\left(G_{x}\left(\eta_{r n}\right)-\lambda\right)\right| \\
& \left.d\left|G_{x_{n}(t)}(v)-G_{x}(v)\right| d t\right],
\end{aligned}
$$

where $x_{n}(t)=F^{-1}\left(F(x)-a_{n} t\right)$ and $V_{n}^{(j, 0)}(t, s)=W_{n}^{(j .0)}(t, s) / W_{n}^{*(j)}(t)$. Now by using integration by parts for the inside integral in the first term of (2.15), the assumption A.III(v) and the boundedness of $W_{n}, W_{n}^{*}$ and $g_{x}$, it follows easily that the first two terms in (2.15) are, uniformly in $r, O\left(\tau_{n}^{2}\right)$; also by using Taylor's expansion and the preceding boundedness assumptions, it follows that the integral in the last term in (2.15) is $O\left(a_{n}\right)$ uniformly in $r$, as $n \rightarrow \infty$. In view of the preceding considerations and the assumption A.III(iv), we obtain from (2.15) that, as $n \rightarrow \infty, \varepsilon_{n 21}=O\left(\tau_{n}^{3 / 2}\right)$ with probability one. This coupled with $(2.14)$ yields

$$
\varepsilon_{n 2}=O\left(\tau_{n}^{3 / 2}\right)
$$

uniformly in $r$, as $n \rightarrow \infty$. Now to deal with the first term, we follow steps similar to those in the proof of Theorem 3.1 (a) of MRR[5]:

Now $\varepsilon_{n 1}=n^{-1} \sum_{i=1}^{n} Z_{n i}^{*}$, with

$$
Z_{n i}^{*}=a_{n}^{-1}\left[W_{n}\left(\left(F(x)-F\left(X_{i}\right)\right) / a_{n}, \eta_{r n}-Y_{i}\right)-W_{n}\left(\left(F(x)-F\left(X_{i}\right)\right) / a_{n}, q_{x}(\lambda)-Y_{i}\right)\right.
$$




$$
\left.-W_{n}^{*}\left(\left(F(x)-F\left(X_{i}\right)\right) / a_{n}\right)\left(G_{x}\left(\eta_{r n}\right)-\lambda\right)\right],
$$

so that using transformation $F(x)-F(u)=a_{n 2}$ and integration by parts in below, we obtain (for notational convenience, we have set $q_{x}=q_{x}(\lambda)$ )

$$
\begin{aligned}
E\left[Z_{n 1}^{*}\right]= & \iint\left[W_{n}\left(t, q_{x}-v+r \tau_{n}^{*}\right)-W_{n}\left(t, q_{x}-v\right)-W_{n}^{*}(t)\left(G_{x}\left(q_{x}+r \tau_{n}^{*}\right)\right.\right. \\
& \left.\left.-G_{x}\left(q_{x}\right)\right)\right] d G_{x_{n}(t)}(v) d t \\
= & \int\left[\int\left(W_{n}\left(t, q_{x}-v+r \tau_{n}^{*}\right)-W_{n}\left(t, q_{x}-v\right)\right) d G_{x_{n}(t)}(v)\right] d t \\
& -\left[G_{x}\left(q_{x}+r \tau_{n}^{*}\right)-G_{x}\left(q_{x}\right)\right] \\
= & \int\left[\int\left[G_{x_{n}(t)}\left(q_{x}-v+r \tau_{n}^{*}\right)-G_{x_{n}(t)}\left(q_{x}-v\right)\right] d W_{n}(t, v)\right] d t \\
& -\left[G_{x}\left(q_{x}+r \tau_{n}^{*}\right)-G_{x}\left(q_{x}\right)\right] \\
= & \left.r \tau_{n}^{*}\right]\left[\int\left\{g_{x_{n}(t)}\left(q_{x}-v\right)-g_{x}\left(q_{x}\right)\right\} d W_{n}^{*}(t, v)\right] d t+O\left(r^{2} \tau_{n}^{* 2}\right)
\end{aligned}
$$

where in (2.18) we have used the boundedness of $g_{x}^{\prime}$ and the proximity of $x_{n}(t)=$ $F^{-1}\left(F(x)-a_{n} t\right)$ to $x$ for $-1 \leq t \leq 1$ and $n$ sufficiently large. Further, employing Taylor's expansion for $x_{n}(t)$ for $n$ sufficiently large and using assumptions A.I, we obtain from (2.18) that for some constant $C_{8}>0$

$$
\begin{aligned}
\left|E\left(Z_{n 1}^{*}\right)\right| & \leq C_{8} \tau_{n}\left|\int\left[g_{x}\left(q_{x}-v\right)-g_{x}\left(q_{x}\right)\right] d m_{n}^{*}(v)\right|+O\left(\tau_{n} a_{n}\right)+O\left(\tau_{n}^{2}\right) \\
& =\sigma\left(\tau_{n}^{3 / 2}\right)
\end{aligned}
$$

as $n \rightarrow \infty$, in view of again the boundedness of $g_{x}^{\prime}$, arguments similar to those of (3.14) to (3.16) of MRR[5] and the assumption A.III(iv), which implies that $a_{n}=$ $\tau_{n}^{\frac{1}{2}}\left[n a_{n}^{5}\left(\log a_{n}^{-1}\right)^{-1}\right]^{1 / 4}=\sigma\left(\tau_{n}^{\frac{1}{2}}\right)$, provided $n a_{n}^{5}$ stays bounded as $n \rightarrow x$. Also similar calculations yield

$$
\begin{aligned}
a_{n} E Z_{n 1}^{* 2}= & \iint\left[W_{n}\left(t, q_{x}-v+r \tau_{n}^{*}\right)-W_{n}\left(t, q_{x}-v\right)\right. \\
& -W_{n}^{*}(t)\left(G_{x}\left(q_{x}+r \tau_{n}^{*}\right)-G_{x}\left(q_{x}\right)\right]^{2} d G_{x_{n}(t)}(v) d t \\
& =\int\left\{\int \left[\left(W_{n}\left(t, q_{x}-v+r \tau_{n}^{*}\right)-W_{n}\left(t, q_{x}-v\right)\right)^{2}\right.\right. \\
& +W_{n}^{* 2}(t) q_{x}^{2}\left(q_{x}+\delta_{n} r \tau_{n}^{*}\right) r^{2} \tau_{n}^{* 2} \\
- & \left.-2 W_{n}^{*}(t) g_{x}\left(q_{x}+\delta_{n} r \tau_{n}^{*}\right) r \tau_{n}^{*}\left(W_{n}\left(t, q_{x}-v+r \tau_{n}^{*}\right)-W_{n}\left(t, q_{x}-v\right)\right)\right] \\
& \left.d G_{x_{n}(t)}(v)\right\} d t \\
\leq & \int\left[\int\left(W_{n}\left(t, q_{x}-v+r \tau_{n}^{*}\right)-W_{n}\left(t, q_{x}-v\right)\right)^{2} d G_{x_{n}(t)}(v)\right] d t
\end{aligned}
$$




$$
\begin{aligned}
& +\left(\int W_{n}^{* 2}(t) d t\right) r^{2} \tau_{n}^{* 2} g_{x}^{2}\left(q_{x}+\delta_{n} r \tau_{\mathbf{n}}^{*}\right) \\
& +2|r| \tau_{n}^{*} g_{x}\left(q_{x}+\delta_{n} r \tau_{n}^{*}\right) \int W_{n}^{*}(t) \mid \int\left[W_{n}\left(t, q_{x}-v+r \tau_{n}^{*}\right)\right. \\
& \left.-W_{n}\left(t, q_{x}-v\right)\right] d G_{x_{n}(t)}(v) \mid d t \\
& \leq \int\left[\int\left(W_{n}\left(t, q_{x}-v+r \tau_{n}^{*}\right)-W_{n}\left(t, q_{x}-v\right)\right)^{2} d G_{x_{n}(t)}(v)\right] d t \\
& +O\left(\tau_{n}\right)
\end{aligned}
$$

where the order term holds uniformly in $r$, as $n \rightarrow \infty$. Now assume momentarily that $r$ $\geq 0$; then the integral within the square bracket in the last R.H.S. expression in $(2.20)$ does not exceed a constant times

$$
\begin{aligned}
& \int\left[W_{n}\left(t, q_{x}-v+r \tau_{n}^{*}\right)-W_{n}\left(t, q_{x}-v\right)\right] d G_{x_{n}(t)}(v) \\
& \quad=\int\left[G_{x_{n}(t)}\left(q_{x}-v+r \tau_{n}^{*}\right)-G_{x_{n}(t)}\left(q_{x}-v\right)\right] d W_{n}(t, v) \\
& =r \tau_{n}^{*} \int g_{x_{n}(t)}\left(q_{x}-v+\delta_{n} r \tau_{n}^{*}\right) d W_{n}(t, v),
\end{aligned}
$$

where in (2.21), we have used integration by parts and the standard mean value theorem. From (2.20) and (2.21), one immediately obtains for some constant $C_{9}>0$

$$
\begin{aligned}
a_{n} E Z_{n 1}^{* 2} & \leq C_{9} \tau_{n} \int\left[\int d W_{n}(t, v)\right] d t+O\left(\tau_{n}\right) \\
& =O\left(\tau_{n}\right)
\end{aligned}
$$

uniformly in $r$, as $n \rightarrow \infty$. The case $r<0$ can be dealt with in a similar manner. Now using the Bernstein inequality as in Theorem 3.1 of MRR[5], we obtain from (2.22)

$$
\begin{aligned}
P\left[n^{-1}\left|\sum_{i=1}^{n}\left[Z_{n i}^{*}-E Z_{n i}^{*}\right]\right| \geq \varepsilon \tau_{n}^{3 / 2}\right] & \leq 2 \exp \left\{\frac{-\varepsilon^{2} n \tau_{n}^{3}}{C_{1} a_{n}^{-1} \tau_{n}+(2 / 3) a_{n}^{-1} \varepsilon \tau_{n}^{3 / 2}}\right\} \\
& =2 \exp \left\{\frac{-\varepsilon^{2} \log a_{n}^{-1}}{C_{1}+(2 / 3) \varepsilon \tau_{n}^{1 / 2}}\right\}
\end{aligned}
$$

for $\varepsilon>0$ and some positive constant $C_{1}$. Using (2.19) and arguing again as in the paragraph immediately after (3.18) of MRR[5], we obtain from (2.23) that, for some positive constants $C_{2}$,

$$
\begin{aligned}
P\left[\max _{|r| \leq d_{n}}\left|J_{n 1}\left(\eta_{r n}\right)-J_{n 1}\left(q_{x}(\lambda)\right)\right|\right. & \left.\geq \varepsilon \tau_{n}^{3 / 2}\right] \\
& \leq 2 d_{n} n^{-2} \leq C_{2} n^{-7 / 4},
\end{aligned}
$$

which in view of Borel-Cantelli lemma implies that 


$$
\max _{\mid r_{i}=d_{n}}\left|J_{n 1}\left(\eta_{r_{n}}\right)-J_{n 1}\left(q_{x}(\lambda)\right)\right|=O\left(\tau_{n}^{3 / 2}\right)
$$

with probability one, as $n \rightarrow x$. Since clearly, using mean value theorem, $\max \left\{\mid G_{x}\right.$ $\left.\left(\eta_{(r+1) n}\right)-G_{x}\left(\eta_{r n}\right) \mid:-d_{n} \leq r \leq d_{n}\right\}=O\left(\tau_{n}^{3 / 2}\right)$ as $n \rightarrow x,(2.24)$ coupled with (2.10) to (2.12), (2.16) and the fact that $t_{n}(x) \rightarrow 1$, with probability one, as $n \rightarrow x$, establishes (2.8). The proof is complete.

\section{The Main Result}

We shall now state the main result of this paper concerning the almost sure representation of the $\lambda^{\text {th }}$ conditional quantile $G_{n x}^{-1}(\lambda)$ (for fixed $0<\lambda<1$ and $x \in$ $\Lambda(F))$ defined by $(1.2)$.

THEOREM 3.1. Under the assumptions of Lemmas 2.1 and 2.2, for each fixed $\lambda$, $0<\lambda<1$ and $x \in \Lambda(F)$, we have with probability one

$$
\left.\widehat{G}_{n x}^{-1}(\lambda)=G_{x}^{-1}(\lambda)+\left[\lambda-\widehat{G}_{n x}\left(G_{x}^{-1}(\lambda)\right)\right] g_{x}^{-1}(\lambda)\right)+O\left(\tau_{n}^{3 / 2}\right),
$$

as $n \rightarrow \infty$, where $\tau_{n}=n^{-\frac{1}{2}} a_{n}^{-\frac{1}{2}}\left(\log a_{n}^{-1}\right)^{\frac{1}{2}}$.

Proof. From Lemmas 2.1 and 2.2, we have at once

$$
\widehat{G}_{n x .}\left(\widehat{G}_{n \cdot x}^{-1}(\lambda)\right)-\widehat{G}_{n \cdot x}\left(G_{x}^{-1}(\lambda)\right) \underset{\text { i... }}{=}\left(\widehat{G}_{n \cdot x}^{-1}(\lambda)-G_{x}^{-1}(\lambda)\right) g_{x}\left(G_{x}^{-1}(\lambda)\right)+O\left(\tau_{n}^{2}\right)+O\left(\tau_{n}^{3 / 2}\right)
$$

where $\tau_{n}=n^{-\frac{1}{2}} a_{n}^{-\frac{1}{2}}\left(\log a_{n}^{-1}\right)^{\frac{1}{2}}$, as $n \rightarrow \infty$, which yields (3.1) by rearrangement of the equality. The proof is complete

The following corollary is an immediate consequence of Theorem 3.1 above and Theorem 3.1 of MRR[5].

CoROLlaRY 3.1. Under the conditions of Theorem 3.1 above, $\widehat{G}_{n x}^{-1}(\lambda)$ is asymptotically normal i.e., for each $\lambda, 0<\lambda<1,\left(n a_{n}\right)^{1 / 2}\left[\widehat{G}_{n x}^{-1}(\lambda)-G_{x}^{-1}(\lambda)\right] \longrightarrow N\left(0, \sigma_{x}^{2}(\lambda)\right)$ $\left(\right.$ or, $N\left(b_{x}\left(G_{x}^{-1}(\lambda)\right), \sigma_{x}^{2}(\lambda)\right)$ if $\left.n a_{n}^{2 m+3} \rightarrow \theta\right)$ as $n \rightarrow \infty$, where $\sigma_{x}^{2}(\lambda)=\lambda(1-\lambda)\left(\int k_{1}^{2}(t) d t\right)$ / $g_{x}^{2}\left(G_{x}^{-1}(\lambda)\right)$ with the kernel function $k_{1}, b_{x}(\cdot)$ and $\theta$ as given in Corollary 3.1 of MRR[5].

REMARK 3.1. It is worth noting that in our proof we have made no assumption regarding continuity or differentiability of the function $W_{n}(t, s)$ in the second argument. Consequently the assertions of Theorems 3.1 and Corollary 3.1 also cover the "unsmoothed" conditional empirical distribution and quantile functions considered by Stute [6] and Härdle et al. [2]. Further, the considerations of Remark 3.3 of MRR[5] will also apply here.

Remark 3.2. The results of Lemmas 2.1 and 2.2, Theorem 3.1 and Corollary 3.1 concerning the RNN estimator $\widehat{G}_{n x}^{-1}(\lambda)$ also remain valid, under the same conditions, for the Nadaraya-Watson type estimator $\widehat{G}_{n x}^{*-1}(\lambda)$ of $G_{x}^{-1}(\lambda), 0<\lambda<1$ defined in Section 1 of MRR[5]. The proofs of these results for the Nadaraya-Watson type estimators - both smoothed and unsmoothed - are in fact contained within the 
corresponding ones for the RNN estimators. This remark covers the assertions of Remark 3.1 as well.

\section{References}

[1] Bahndur, R.R.: A note on quantiles in large samples, Ann. Math. Statist., 37 (1966). 577-580.

[2] Härdle, W., Janssen. P. and Serfling R.: Strong uniform convergence rates for estimation of conditional functionals. Ann. Statist., 16 (1988), 1428-1449.

[3] Kiefer, J.: On Bahadur's representation of sample quantiles, Ann. Math. Statist. 38 (1967), 13231342 .

[4] MAck, Y.P.: Bahadur's representation of sample quantiles based on smoothed estimates of a distribution function. Prob. and Math. Statist., 8 (1987), 183-189.

[5] Mehrs, K.L., Rama Krishnatah, Y.S. and Rao, M. SudhnkirA: Asymptotic properties of smoothed is. unsmoothed conditional distribution function estimators, Bulletin of Informatics and Cybernetics, 25 (1992) $(1-2), 71-97$.

[6] Stute, W.: Conditional empirical processes, Ann. Statist., 14 (1986), 638-647.

Received June 21, 1990

Revised June 11, 1991

Communicated by H. Yamato 